\title{
Calidad de vida laboral en personal de enfermería de un hospital privado en Yucatán
}

Quality of work life in nursing staff at a private hospital in Yucatán

Qualidade de vida no trabalho em pessoal de enfermagem de um hospital privado em Yucatan

Ruth Noemí Ojeda López Universidad Autónoma de Yucatán, México ruth.ojeda@correo.uady.mx https://orcid.org/0000-0002-7137-120X

Jennifer Mul Encalada Universidad Autónoma de Yucatán, México jeni.mul@correo.uady.mx https://orcid.org/0000-0003-0034-8165

Olivia Jiménez Díez Universidad Autónoma de Yucatán, México jdiez@correo.uady.mx https://orcid.org/0000-0002-4514-5241 


\section{Resumen}

Desempeñarse en el área de servicios de salud no es fácil, ya que los profesionales que ahí laboran están expuestos a situaciones de crisis y desgaste emocional. El presente trabajo tuvo como objetivo analizar la calidad de vida laboral del personal de enfermería de un hospital privado del estado de Yucatán. La investigación fue con un enfoque cuantitativo, el diseño no experimental, descriptivo de corte transversal y correlacional. La muestra estuvo conformada por la plantilla de personal de enfermería del hospital estudiado, integrada por 84 profesionales, de los cuales 72 fueron mujeres y 12 hombres, con una media aritmética de 33 años, el 51.19\% solteros, en su mayoría con escolaridad de carrera técnica y menos de 10 años de antigüedad laboral. Se les aplicó el instrumento de medición de Calidad de Vida Laboral denominado ProQOL 5. Este instrumento está conformado por tres subescalas, satisfacción por compasión (SC), síndrome de quemado (SQ) y estrés por trauma secundario (ETS), correspondiendo 10 reactivos a cada una de ellas, con medición de tipo Likert de 5 puntos siendo: 1 = Nunca, $2=$ Rara vez, $3=$ Alguna vez, $4=$ A menudo y $5=$ Frecuentemente. En estudio previo en México, Mendoza, García y Serna (2014) realizaron análisis factorial confirmatorio y modelos de ecuaciones estructurales para su validación obteniendo un coeficiente de alfa de Cronbach de .75 .

En general los resultados señalan que un importante número del personal se ubica en niveles altos de satisfacción por compasión y bajos en síndrome de quemado y estrés por trauma secundario. De las variables sociodemográficas estudiadas únicamente la escolaridad resultó ser un factor diferenciador en relación con el síndrome de quemado. Así mismo se encontró, concordante con la literatura, que el síndrome de quemado tiene una relación directa con el estrés por trauma secundario, aunque muy baja en el hospital estudiado.

Palabras clave: Calidad de vida laboral, burnout, satisfacción por compasión, estrés, hospital, enfermería. 


\section{Abstract}

Performing in the health services area is not easy, since the professionals who work there are exposed to situations of crisis and emotional exhaustion. The objective of this work was to analyze the quality of work life of the nursing staff of a private hospital in the state of Yucatan. The research was done with a quantitative approach, non-experimental, descriptive cross-sectional and correlational design. The sample was made up of the nursing staff of the studied hospital, participating 84 professionals, of whom 72 were women and 12 men, with an arithmetic mean of 33 years old, $51.19 \%$ single, the majority with technical career education level and less than 10 years at work. The Work Quality of Life measurement instrument called ProQOL 5 was applied to them. This instrument is made up of three subscales, compassion satisfaction (CS), burn syndrome (BO) and secondary trauma stress (STS), corresponding to 10 items each one, with a 5-point Likert-type measurement, being: $1=$ Never, $2=$ Rarely, $3=$ Sometimes, $4=$ Often y $5=$ Very often.

In a previous study in Mexico, Mendoza, García and Serna (2014) carried out confirmatory factor analysis and structural equation models for validation, obtaining a Cronbach's alpha coefficient of .75.

In general, the results indicate that a significant number of staff are located at high levels of satisfaction due to compassion and low levels of burn syndrome and stress from secondary trauma. Of the sociodemographic variables studied, only schooling level turned out to be a differentiating factor in relation to burn syndrome. Likewise, it was found, consistent with the literature, that burn syndrome has a direct relationship with stress from secondary trauma, although very low in the hospital studied.

Keywords: Quality of work life, burnout, satisfaction with compassion, stress, hospital, nursing. 


\section{Resumo}

Atuar na área de serviços de saúde não é fácil, pois os profissionais que trabalham lá estão expostos a situações de crise e exaustão emocional. $\mathrm{O}$ objetivo deste trabalho foi analisar a qualidade de vida no trabalho da equipe de enfermagem de um hospital privado do estado de Yucatán. A pesquisa foi realizada com abordagem quantitativa, não experimental, descritiva, de corte transversal e correlacional. A amostra foi composta pela equipe de enfermagem do hospital estudado, composta por 84 profissionais, sendo 72 mulheres e 12 homens, com média aritmética de 33 anos, 51,19\% solteiros, a maioria com formação profissional. técnico e com menos de 10 anos de idade. Aplicou-se o instrumento de medida de Qualidade de Vida no Trabalho denominado ProQOL 5. Esse instrumento é composto por três subescalas, satisfação com compaixão (SC), síndrome de queimadura (SQ) e estresse secundário ao trauma (ETS), correspondendo a 10 itens a serem cada um, com uma medição do tipo Likert de 5 pontos, sendo: $1=$ Nunca, $2=$ Raramente, $3=$ Algum dia, $4=$ Frequentemente $\mathrm{e} 5=$ Frequentemente.

Em um estudo anterior no México, Mendoza, García e Serna (2014) realizaram análise fatorial confirmatória e modelos de equações estruturais para validação, obtendo um coeficiente alfa de Cronbach de 0,75.

Em geral, os resultados indicam que um número significativo de funcionários está localizado em altos níveis de satisfação devido à compaixão e baixos níveis de síndrome de queimadura e estresse secundário ao trauma. Das variáveis sociodemográficas estudadas, apenas a escolaridade se mostrou um fator diferenciador em relação à síndrome de queimadura. Da mesma forma, verificou-se, de acordo com a literatura, que a síndrome da queimadura tem uma relação direta com o estresse secundário do trauma, embora seja muito baixa no hospital estudado.

Palavras-chave: Qualidade de vida no trabalho, desgaste, satisfação por compaixão, estresse, hospital, enfermagem.

Fecha Recepción: Junio 2019

Fecha Aceptación: Diciembre 2019 


\section{Introducción}

Es un hecho que las personas que laboran pasan aproximadamente una tercera parte de su tiempo en el lugar de trabajo, por ello la salud de los empleados es requisito indispensable tanto para la estabilidad familiar como para la productividad y desarrollo económico de las organizaciones. Por lo que, contar con condiciones de trabajo adecuadas, proporciona oportunidades de desarrollo personal, protección contra riesgos físicos y psicosociales. Aunado a lo anterior, puede también mejorar las relaciones sociales, la autoestima de los trabajadores y repercutir positivamente en su salud (Secretaría del Trabajo y Previsión Social, STPS, 2017).

De acuerdo con Segurado y Agulló (2002), estudiar la calidad de vida laboral permite identificar áreas de mejora de las condiciones laborales y disminuir las afectaciones psicosociales de los trabajadores.

En el caso de las organizaciones de salud, Sibbald, Enzer, Cooper, Rout y Sutherland (2000) señalan que la calidad de los servicios prestados en ellas se relaciona directamente con la satisfacción y la calidad de vida laboral de los profesionales que la integran, por lo que éstos representan su principal activo.

En particular el personal de enfermería colabora en la promoción de la salud, atención a los enfermos en diferentes situaciones en que se encuentren, ya sea situaciones leves como críticas e incluso terminales, trabajando de manera autónoma o en colaboración (Organización Mundial de la Salud, OMS, 2015). Estas particularidades de sus actividades hacen que su calidad de vida pueda verse afectada. Desde 2015, la misma OMS señalaba que la enfermería, es una profesión que tendría cada vez mayor relevancia en la respuesta de los sistemas de salud, ante las condiciones epidemiológicas futuras de las poblaciones, lo cual actualmente se ha hecho realidad con la pandemia del Covid-19.

Dada su importancia tanto a nivel familiar como organizacional se justifica la realización de diversos estudios que, como en este caso, exploren o profundicen en su conocimiento. Debido a lo anterior, el presente estudio tuvo como objetivo analizar la calidad de vida laboral del personal de enfermería de un hospital privado del estado de Yucatán. 


\section{Desarrollo}

\section{Aspectos conceptuales}

De acuerdo con el Grupo Calidad de Vida de la Organización Mundial de la Salud (WHOQOL, por sus siglas en inglés) se entiende por calidad de vida "las percepciones individuales acerca de su posición en la vida en el contexto de la cultura y los sistemas de valores en los cuales se encuentran viviendo en relación con sus metas, expectativas, estándares y preocupaciones" (WHOQOL, 1998, p.551). Dicha definición presenta la complejidad del término calidad de vida ya que considera el contexto cultural, social y medioambiental, mismos que difieren en las diferentes esferas económico, socioculturales, y entre los individuos mismos.

Según la Fundación Europea para el Mejoramiento de las Condiciones de Vida (2002), la calidad de vida laboral es un constructo multidimensional, hecho de factores interrelacionados, que se asocia con la satisfacción laboral, el involucramiento laboral, seguridad en el trabajo, productividad, salud, desarrollo de competencias, habilidades profesionales y el balance entre la vida laboral y no laboral de los trabajadores. De tal manera que, para que un trabajador tenga un rendimiento adecuado, debe no solo contar con los instrumentos de trabajo correspondientes, sino con las condiciones óptimas y que sean de utilidad en su profesión.

Los trabajadores del sector salud proveen asistencia y servicios a diferentes pacientes y clientes dentro de ambientes laborales potencialmente estresantes. De acuerdo con Hanzeliková, García, Pomares, Pardo y Del Monte (2011), la calidad de los servicios prestados en las organizaciones sanitarias se relaciona directamente con la satisfacción de los profesionales que las integran, así como con otros factores como los accidentes laborales, reacciones negativas en la actitud y desempeño. Aun cuando estos profesionales cuentan con un entrenamiento son especialmente susceptibles a experimentar respuestas traumáticas en ellos mismos (Lloyd, King y Chenoweth, 2002).

Los resultados de distintas investigaciones muestran que, factores como el síndrome de quemado o burnout, están directamente relacionados con el nivel de calidad de vida laboral; de acuerdo con Flores, Jenaro, Cruz, Vega, y Pérez (2013), los profesionales de enfermería presentan mayor riesgo de burnout además de ser quienes presentan mayores afectaciones en su salud física. 
Revista Iberoamericana

de las Ciencias Sociales y

Humanísticas

ISSN: $2395-7972$

Dentro de los resultados de Díaz, Stimolo y Caro, (2010) se tiene que aquellos profesionales de enfermería que presentan un nivel medio de burnout aun presentan una alta realización personal. Otro factor relacionado con la calidad de vida laboral es la satisfacción por compasión, específicamente en los profesionales de la salud, es este factor el que coloca al profesional cerca del enfermo, lo conecta y facilita que gestione sus sentimientos, lo cual disminuye el burnout; sin embargo, aquellos profesionales que viven el cuidado con desgaste y sin profesionalismo, no logran cercanía con el enfermo y deshumanizan su atención (Buceta, Bermejo y Villacieros, 2019).

De acuerdo con Marín-Tejeda (2017), el burnout es un concepto ampliamente estudiando en cuanto al desgaste profesional, y la fatiga por compasión (FC) es más bien un concepto que todavía se está estudiando debido a ser reciente, sin embargo, ambos están vinculados con la prestación de servicios asistenciales y el efecto negativo que puede tener en los prestadores de servicios. Los resultados de su investigación demuestran que los trabajadores mexicanos no tienen problema en demostrar agotamiento emocional, más no así síntomas de cinismo, y que aunque están sometidos a un fuerte nivel de estrés, muestran alto grado de satisfacción profesional, lo cual se corrobora con las altas puntuaciones de satisfacción por compasión (SC), y que concuerda con lo establecido por Stamn (2010) en cuanto a que la satisfacción por compasión constituye un factor protector contra el desgaste.

El estrés traumático secundario (ETS) es otro factor relacionado con la calidad de vida laboral, ya que muchos profesionales de la salud son propensos a padecerlo, debido a la naturaleza propia de su profesión, ya que son ellos quienes se preocupan y se relacionan de manera cercana con los pacientes. De acuerdo con Moreno, Morante, Rodríguez y Garrosa (como se cita en Meda, Moreno-Jiménez, Palomera, Arias, y Vargas, 2012), se entiende por ETS el proceso por el cual el individuo que asiste a otra persona (paciente), experimenta de forma paralela las mismas respuestas emocionales que la otra persona está experimentando. Según Figley (citado en Meda et al., 2012) existen cuatro factores que influyen en la predisposición a experimentar ETS, la empatía, el comportamiento hacia la víctima, la habilidad para distanciarse del trabajo y el propio sentimiento de satisfacción por ofrecer ayuda. 


\section{Revista Iberoamericana \\ de las Ciencias Sociales y Humanísticas}

ISSN: $2395-7972$

De acuerdo con lo anteriormente mencionado se identifican reacciones psicológicas que los profesionales de la salud pueden experimentar como consecuencia de su trabajo con pacientes: la satisfacción por compasión, el síndrome del quemado, la fatiga por compasión y el estrés traumático secundario. Para efectos del trabajo que se presenta se decidió emplear el Modelo de Calidad de Vida Profesional propuesto por Stamm (2010), en este modelo se entiende por satisfacción por compasión, los aspectos positivos que se tienen dentro del desempeño como profesional de la salud; y los aspectos negativos del mismo desempeño se les conoce como el desgaste o fatiga por compasión. La fatiga por compasión se divide en dos elementos: la primera se ocupa de situaciones como el agotamiento, la frustración, la rabia y depresión, mejor conocido como burnout. Y la segunda se refiere al estrés traumático secundario, lo cual se refiere a un sentimiento negativo provocado por la relación de ayuda y empatía hacia personas que han sufrido un trauma (Ver figura 1).

Figura 1. Modelo de Calidad de Vida Profesional.

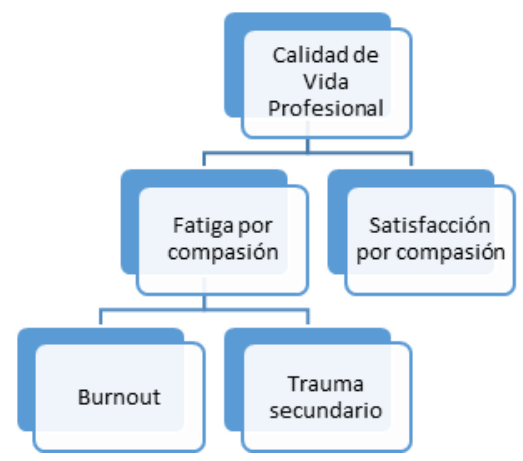

Fuente: Traducción libre de Stamm (2010).

El burnout ha estado relacionado con condiciones laborales estresantes, traumatización vicaria $^{1}$ y a la falta de recursos y apoyo. Es un hecho que, si no se cuentan con estrategias efectivas para manejar el estrés y el burnout, la satisfacción global con el trabajo y el ayudar a otros -satisfacción por compasión- puede estar comprometida y no lograrse (Stamm, 2010).

\footnotetext{
${ }^{1}$ De acuerdo con Armayones (2010, p.215) "Es el efecto de experimentar, vivenciar, de vivir la angustia, el dolor, el miedo y cualquier otra emoción negativa de las que viven las personas a las que se está ayudando, es la exposición secundaria al trauma y la implicación emocional a las experiencias traumáticas de los usuarios".
} 
Se entiende por trauma secundario, la exposición a eventos traumáticos experimentados mientras se escucha a otros contar sus propias historias (Pryce, Shackelford y Pryce, 2007), y son precisamente estos eventos traumáticos los que contribuyen al síndrome del quemado en detrimento de la salud mental del trabajador.

El concepto global de calidad de vida profesional es complejo ya que involucra tanto las características del medio ambiente laboral, como las características personales del individuo y la exposición del profesional a traumas primarios y secundarios en el medio laboral. Esto aplica tanto a los profesionales pagados como al voluntariado.

La figura 2 ilustra los elementos de la calidad de vida profesional. Como se puede observar en el centro de la figura están la satisfacción por compasión y la fatiga por compasión. De igual forma se observa cómo el medio ambiente del paciente (o persona a la cual se le está ayudando) y el ambiente del propio profesional (el que ayuda) tiene un rol. Por ejemplo, un pobre ambiente laboral puede contribuir al concepto de fatiga por compasión, pero al mismo tiempo la persona puede sentir satisfacción por compasión por el hecho de poder ayudar a otros a pesar del pobre ambiente laboral. La fatiga por compasión contiene dos aspectos que, aunque son muy diferentes ambos son negativos, el trauma relacionado con el trabajo tiene el elemento miedo como un aspecto distintivo asociado a él, y cuyo efecto en la persona es mucho más fuerte que el burnout. La figura 2 muestra el análisis teórico de los resultados positivos y negativos de ayudar a otros que han experimentado estrés traumático. 


\section{Revista lberoamericana de las Ciencias Sociales y Humanísticas}

ISSN: $2395-7972$

Figura 2. Análisis teórico de resultados sobre la Calidad de Vida Profesional.



Fuente: Traducción libre de Stamm (2010).

Para un mejor entendimiento del Modelo de Calidad de Vida Profesional, a continuación, se describen los elementos esenciales del mismo.

La satisfacción por compasión de acuerdo con Stamm (2010, p.8) "son los sentimientos positivos que las personas experimentan resultado de su habilidad de ayudar a otros" y se relaciona con el concepto de calidad de vida laboral. Contrario al síndrome del quemado o al estrés traumático secundario, el trabajador obtiene satisfacción por compasión a través del sentido de logro de su labor, de la motivación continua o aún de su propia inspiración y disfrute que le proporciona el demandante trabajo social que significa lidiar con personas en casos de crisis. Por ejemplo, el agradecimiento de las personas que reciben la ayuda, el sentimiento del deber cumplido, la sensación de que el trabajo tiene utilidad y trascendencia para las personas que se atienden, para la organización, la profesión o la sociedad en general. Por ello se dice que la satisfacción por compasión es un medio efectivo para reducir el burnout y /o ETS ya que provee de motivación, interés y sentido de logro cuando se ayuda a los pacientes a sobrepasar traumas (Bride, Radey y Figley, 2007).

También tiene que ver con las buenas relaciones con los compañeros, buenas condiciones ambientales del trabajo, el reconocimiento profesional, etc. La satisfacción por compasión origina situaciones en las cuales los profesionales se benefician colateralmente cuando sus 


\section{Revista Iberoamericana de las Ciencias Sociales y Humanísticas}

ISSN: $2395-7972$

pacientes comparten su mejoramiento funcional, crecimiento personal y/o mejoras terapéuticas (Pooler, Wolfer y Freeman, 2014). De manera que se puede observar más fácilmente en mayor rendimiento, actitud positiva hacia el trabajo, valoración mejorada y una mayor esperanza por resultados positivos que se visualizan entre los profesionales exitosos (Kulkarni, 2013). La satisfacción por compasión se puede ver como antitética al concepto de fatiga por compasión, debido a que el agotamiento o la desesperación se apoderan del trabajo de uno, lo que lleva al agotamiento (Stamm, 2010). Sin embargo, no se conoce ninguna fórmula o enfoque simplificado para la satisfacción por compasión Aquellos que obtienen una calificación alta en cuanto la satisfacción por compasión en realidad muestran su habilidad para ser cuidadores efectivos en su trabajo.

Figley (1995) fue quien originalmente conceptualizó la fatiga por compasión para describir los efectos acumulativos de trabajar con individuos que han experimentado traumas que generan estrés traumático secundario. La fatiga por compasión se subdivide en dos partes, la primera se relaciona con situaciones de frustración, agotamiento, coraje, y depresión, todos típicos elementos del burnout. Y la segunda parte tiene que ver con el estrés traumático secundario. Es importante recordar que algunos traumas laborales pueden ser traumas directos o primarios o que pueden ser una combinación de traumas primarios y secundarios. La fatiga por compasión es resultado de la exposición del profesional al material traumático de los pacientes en combinación con su sentido de empatía por el paciente. De tal forma que el nivel de empatía del profesional incrementa el riesgo de experimentar fatiga por compasión. Así pues, a mayor empatía por parte del profesional, mayor el riesgo de padecer fatiga por compasión. Dado que la fatiga por compasión representa una reacción natural por trabajar en ambientes que despliegan crisis, pudiera ser considerado un riesgo laboral (Munroe, 1999). Sin embargo, hay que reconocer que la fatiga por compasión es un riesgo laboral tiene serias implicaciones para la salud mental y el campo laboral.

A finales de la década de los 70's comenzaron a hacerse estudios con profesiones relacionadas con la atención de pacientes, y es ahí donde se comienza a hablar del concepto de calidad de vida laboral y se le relaciona con el burnout o síndrome del quemado, como resultado de una prolongada prestación de servicios y cuidado de personas con problemas de salud (Hurtado y Pereira, 2012). El burnout es un elemento de los efectos negativos del cuidado que se conoce como fatiga por compasión. La mayoría de la gente tiene una idea 


\section{Revista Iberoamericana de las Ciencias Sociales y Humanísticas}

ISSN: $2395-7972$

intuitiva de lo que es el burnout. Desde la perspectiva de la investigación, el burnout se asocia con sentimientos de desesperanza y dificultades para lidiar con el trabajo o para hacer su trabajo de manera efectiva. Estos sentimientos negativos generalmente tienen un inicio gradual. Pueden reflejar la sensación de que sus esfuerzos no hacen la diferencia, o puede ser asociado con una carga de trabajo muy alta o un entorno de trabajo no solidario (Stamm, 2010).

A lo anterior se puede sumar el burnout profesional debido a los obstáculos burocráticos del día a día, la excesiva carga de trabajo y el mal funcionamiento de la organización. De acuerdo con el estudio internacional de Dall'Occhio y Lermoli, (2012, p.4), se estima que uno de cada cuatro profesionales del servicio hospitalario (enfermeras, médicos, rehabilitadores) están en riesgo de padecer burnout. "Más del 30 \% de los médicos argentinos padecen el síndrome de burnout". Argentina junto con México y Colombia son los países que encabezan la lista de países con mayor cantidad de médicos con burnout.

De acuerdo con Castañeda y García del Alba (citados en Aranda, Pando y Salazar, 2015) el 41.6\% de los médicos familiares mexicanos padecen de burnout, además de que existen diferencias significativas en relación con el sexo, edad, antigüedad, tipo de contratación entre otros. Los resultados de Aranda et al. (2015) muestran que el desgaste ocupacional de los mexicanos se relacionan con las variables demográficas como el sexo, estado civil, escolaridad, tener hijos y las edades de los mismos, así como con variables relacionadas al tipo de trabajo que realizan y la toma de decisiones; de tal forma que las mujeres presentan mayor tendencia, así como aquellos que tienen hijos pequeños, baja escolaridad, se desempeñan en trabajos operativos pero que tienen bajo control en la toma de decisiones, y se encuentran en una etapa de desarrollo y adaptación laboral.

Lo anterior se reafirma con los resultados de Ansoleaga, Vézina y Montañon (citados en Aranda et al., 2015), ya que de acuerdo con sus resultados las mujeres son las más propensas a la depresión debido a que reciben bajas recompensas, aunque los varones también la sufren, pero por las demandas psicológicas y por los desequilibrios entre esfuerzo y recompensas. De acuerdo con Villavicencio-Ayub (2019), el 35\% de los mexicanos entre 29 y 48 años son considerados adictos al trabajo, así se constata en las estadísticas de la Organización para la Cooperación y el Desarrollo Económico (OCDE, 2019) que muestran que México es el país que tiene el mayor número de horas laborales al año (2,257 horas anuales de trabajo), lo cual 
Revista Iberoamericana

de las Ciencias Sociales y

Humanísticas

ISSN: $2395-7972$

sobrepasa por mucho la media de la OCDE (1,744 hrs). En cuanto al burnout el $40 \%$ de los mexicanos lo padece presentando entre otros síntomas el agotamiento físico y emocional, indiferencia y frustración entre otros.

El estrés traumático secundario es un elemento de la fatiga por compasión, se relaciona con el trabajo y con la exposición secundaria a las experiencias extremadamente traumáticas de eventos experimentados por otras personas (pacientes). Algunos de sus efectos negativos incluyen miedo, dificultades para dormir e imágenes intrusivas recordatorias de experiencias traumáticas. El estrés traumático secundario se relaciona con el trauma vicario, ya que tienen muchas características similares (Stamm, 2010).

Adicionalmente al modelo propuesto se consideró la teoría de conservación de recursos Hobfoll (1998) que en sus inicios buscaba explicar las reacciones de afrontamiento del estrés, proponiendo posteriormente el principio de que "los individuos se esfuerzan para obtener, mantener y proteger las cosas que valoran" (Hobfoll, 2001, p. 337).

\section{Metodología}

La investigación fue con un enfoque cuantitativo, con diseño no experimental, descriptivo de corte transversal y correlacional, ya que tuvo como propósito mostrar o examinar la relación entre variables, pero no relaciones causales (Bernal, 2010).

Se administró un cuestionario y participaron voluntariamente 84 personas que integraban todo el personal de enfermería de un hospital privado del Estado de Yucatán, de los cuales 72 fueron mujeres y 12 hombres, equivalente en términos porcentuales a $85.7 \%$ de mujeres y $14.3 \%$ de hombres. Puede observarse que la mayor parte de los profesionales son jóvenes, al encontrarse en el rango de 20 a 29 años, sin embargo, poco más del $24 \%$ de la muestra tenía 40 o más años. La media aritmética de la edad es de 33 años.

De los sujetos estaban casados 39.29\%; viviendo en unión libre el 9.52\%, mientras que los solteros, divorciados, separados y viudos representaron el $51.19 \%$ de la muestra.

Se observa que casi dos terceras partes de los profesionales en enfermería tienen la carrera técnica en enfermería (69.05\%), mientras que la licenciatura en enfermería representa casi la tercera parte restante $(30.95 \%)$ de la muestra.

Los años de antigüedad laborando como profesional en enfermería estuvo entre 0 a 44 años, sin embargo, predomina gente con poca antigüedad considerando el rango de 0 a 10 años que 
Revista Iberoamericana

de las Ciencias Sociales y

Humanísticas

ISSN: $2395-7972$

representó el 53.01\% de la muestra; entre 11 a 20 años el 17.86\%; entre 21 a 30 años el $17.86 \%$ y más de 30 años sólo el $4.76 \%$.

En cuanto a los años que piensan seguir trabajando como profesionales de la enfermería presenta una media de 21.47 años con una desviación estándar de 12.10.

El Estado de Yucatán es considerado con potencial para ser un polo de turismo de salud importante en el país, dada su infraestructura hospitalaria. En el gobierno del presidente Fox fue seleccionado para establecer uno de los 6 hospitales regionales de alta especialidad.

El hospital estudiado es un hospital de especialidades médicas con más de 30 años de experiencia, cuenta con alto prestigio entre la sociedad yucateca y su área de influencia abarca la península de Yucatán.

El instrumento de medición aplicado fue el de Calidad de Vida Laboral denominado ProQOL 5 (Professional Quality of Life Scale -ProQOL), el cual es comúnmente utilizado para medir efectos positivos y negativos del trabajo en aquellas personas que han experimentado eventos extremadamente estresantes. Dicho instrumento se compone por tres sub-escalas, satisfacción por compasión, síndrome de quemado y estrés por trauma secundario. En total se integra por 30 reactivos en una escala tipo Likert de cinco puntos en la que $1=$ Nunca, 2 = Rara vez, 3 = Alguna vez, 4 = A menudo y 5 = Frecuentemente. Los puntajes más altos representan mayores niveles en cada una de las subescalas. Las cuales se obtienen a través de la suma de las puntuaciones obtenidas por cada participante en los ítems correspondientes, permitiendo obtener un puntaje por cada subescala, siendo la puntuación mínima de 1 y la máxima de 50. Para su análisis e interpretación global se establecen rangos de referencia, siendo estos: bajo de 1 a 22, medio de 23 a 41 y alto más de 42. A partir de lo cual se cataloga la calidad de vida laboral en cinco niveles:

1) Alta SC, moderado a bajo SQ y ETS (Reforzado positivamente).

2) Alto SQ, moderado a bajo SC y ETS (Riesgo bajo).

3) Alto ETS con bajo SQ y baja SC (Riesgo medio).

4) Alto ETS y alto SC con bajo SQ (Riesgo alto).

5) Alto ETS y alto SQ con bajo SC (En peligro).

En México, Mendoza, García y Serna (2014) realizaron un estudio en el que participó un grupo de 238 profesionales en enfermería de tres instituciones de salud públicas de Baja California e hicieron la validación del instrumento mediante análisis factorial confirmatorio 
y modelos de ecuaciones estructurales. En cuanto a la confiabilidad obtuvieron un coeficiente de alfa de Cronbach de 0.75.

El procesamiento de los datos se llevó a cabo con el software estadístico Statistical Package for the Social Sciences (SPSS) versión 23. Se realizó un análisis descriptivo de los componentes de la CVL, así como análisis inferencial y correlacional.

\section{Resultados}

En este apartado, se presentan los resultados del procesamiento de la información obtenida mediante la aplicación de las encuestas a los profesionales en enfermería. Inicialmente se incluye un análisis descriptivo y posteriormente los análisis inferenciales y de correlación.

\section{Análisis descriptivo}

En este apartado se presenta un análisis de los niveles y estadísticos de las escalas resultado del comportamiento de la variable calidad de vida laboral y de los componentes de ésta: satisfacción por compasión, síndrome de quemado y estrés por trauma secundario.

En la tabla 1 se pueden observar las medidas de tendencia central y dispersión de cada uno de los componentes de la calidad de vida laboral.

Tabla 1. Medidas de tendencia central y dispersión de los componentes de calidad de vida laboral.

\begin{tabular}{|c|c|c|}
\hline Componentes CVL & Media & Desviación estándar \\
\hline Satisfacción por compasión & 43.01 & 4.91 \\
\hline Síndrome de quemado & 20.85 & 3.92 \\
\hline Estrés por trauma secundario & 18.35 & 4.66 \\
\hline
\end{tabular}

Fuente: Elaboración propia.

La satisfacción por compasión fue el componente de la calidad de vida laboral que obtuvo la calificación más elevada $(\overline{\mathrm{x}}=43.01, \mathrm{~s}=4.91)$. Tomando en consideración las respuestas de algunos ítems en particular del instrumento, puede observarse que el $56.0 \%$ de los participantes manifestó que casi siempre sienten satisfacción; el 50.0\% orgullo; el $60.7 \%$ que disfrutan ayudar y el 72.6\% que están felices por la profesión elegida. Sin embargo, en cuanto 


\section{Revista Iberoamericana de las Ciencias Sociales y Humanísticas}

al ítem relacionado con la sensación de poder cambiar las cosas a través de su trabajo, un $36.9 \%$ de la muestra indicó que sólo "algunas veces" es posible.

El síndrome de quemado se identificó principalmente en nivel bajo, los indicadores estadísticos son $\overline{\mathrm{x}}=20.85, \mathrm{~s}=3.92$. Es de resaltar que un alto porcentaje de los participantes manifestó que casi siempre o muy a menudo son felices $(91.7 \%)$, se sienten vinculados a otras personas debido a su trabajo (59.5\%) y que sus creencias religiosas o espirituales le apoyan (70.2\%), de igual modo la mayoría afirmó que algunas veces, a menudo o casi siempre se perciben como demasiado sensibles (56.0\%) y que su trabajo les hace sentir $\operatorname{agotados}(44.1 \%)$.

El estrés por trauma secundario fue el componente de la calidad de vida laboral con la media más baja $(\overline{\mathrm{x}}=18.35, \mathrm{~s}=4.66)$. Cabe hacer mención que la mayoría de los participantes afirmaron que a menudo o en algunas situaciones se sienten preocupados por una o más de las personas que han ayudado (69.1\%), así mismo, mencionó la mayoría que nunca o rara vez han considerado haber sido afectados negativamente (91.7\%), haberse sentido deprimidos por las experiencias traumáticas de los pacientes (90.4\%) o han evitado actividades o situaciones que les recuerdan dichas experiencias traumáticas (89.3\%).

Como se mencionó en la metodología para el análisis e interpretación global se establecen rangos de referencia, siendo estos: bajo de 1 a 22, medio de 23 a 41 y alto más de 42 (Stamm, 2010). Los resultados de las tres subescalas se presentan en la tabla 2.

Tabla 2. Niveles de los componentes de CVL.

\begin{tabular}{|c|c|c|c|}
\hline Nivel & Frecuencia SC & Frecuencia SQ & Frecuencia ETS \\
\hline Bajo & 0 & 60 & 71 \\
\hline Moderado & 31 & 24 & 13 \\
\hline Alto & 53 & 0 & 0 \\
\hline
\end{tabular}

Fuente: Elaboración propia.

Se puede apreciar que la mayoría tiene una alta satisfacción por compasión (63\%) y bajo síndrome de quemado (71\%) y estrés por trauma secundario (86\%).

En la tabla 3 se presenta la distribución de la muestra en cada una de las categorías de calidad de vida laboral establecidas en el ProQOL (Stamm, 2010). Puede observarse que más de la mitad de la muestra se encuentra en la categoría más alta y positiva. 
Revista Iberoamericana

de las Ciencias Sociales y

Humanísticas

ISSN: 2395 - 7972

Es importante considerar que el resto de la muestra no cumplió con las características de alguna de las 4 categorías restantes establecidas en el ProQOL, relacionadas con situaciones de riesgo (alto síndrome de quemado o alto estrés por trauma secundario).

Tabla 3. Distribución de la muestra de acuerdo con las categorías de calidad de vida laboral.

\begin{tabular}{|l|r|r|r|}
\hline \multicolumn{1}{|c|}{ Categorías de CVL } & Mujeres & Hombres & Total \\
\hline $\begin{array}{l}\text { 1. Alta SC + moderado a bajo SQ y ETS } \\
\text { (Reforzado positivamente) }\end{array}$ & $44(52.4 \%)$ & $9(10.7 \%)$ & $53(63.1 \%)$ \\
\hline $\begin{array}{l}\text { 2. Alto SQ + moderado a bajo SC y ETS } \\
\text { (Riesgo bajo) }\end{array}$ & 0 & 0 & 0 \\
\hline $\begin{array}{l}\text { 3. Alto ETS + bajo SQ y bajo SC } \\
\text { (Riesgo medio) }\end{array}$ & 0 & 0 & 0 \\
\hline $\begin{array}{l}\text { 4. Alto ETS y alto SC con bajo SQ } \\
\text { (Riesgo alto) }\end{array}$ & 0 & 0 & 0 \\
\hline $\begin{array}{l}\text { 5. Alto ETS y alto SQ con bajo SC } \\
\text { (En peligro) }\end{array}$ & 0 & 0 & 0 \\
\hline Ninguno de los perfiles & $28(33.3 \%)$ & $3(3.6 \%)$ & $31(36.9 \%)$ \\
\hline
\end{tabular}

Fuente: Elaboración propia

\section{Análisis Inferencial}

A continuación, se presentan los análisis correspondientes a las escalas con relación a los datos sociodemográficos sexo, estado civil y escolaridad.

En la tabla 3, se concentran los resultados de las pruebas t para muestras independientes y de dichos resultados se concluye que ninguna de las variables analizadas presentó diferencias significativas por sexo. 
Revista Iberoamericana

de las Ciencias Sociales y Humanísticas

Tabla 3. Estadísticos por sexo.

\begin{tabular}{|c|c|c|c|}
\hline Factores & Sexo & Media & $\begin{array}{l}\text { Desviación } \\
\text { Estándar }\end{array}$ \\
\hline \multirow{2}{*}{ Satisfacción por compasión } & Hombre & 54.39289 & 8.375716 \\
\hline & Mujer & 49.26785 & 10.110764 \\
\hline \multirow[t]{2}{*}{ Síndrome de quemado } & Hombre & 51.63810 & 11.317711 \\
\hline & Mujer & 49.72698 & 9.824829 \\
\hline \multirow[t]{2}{*}{ Estrés por trauma secundario } & Hombre & 51.22672 & 8.801877 \\
\hline & Mujer & 49.79555 & 10.227503 \\
\hline
\end{tabular}

Fuente: Elaboración propia

Se observa en la tabla 4 que esta variable no es un factor diferenciador para alguna de las escalas.

Tabla 4. Análisis por sexo

\begin{tabular}{|l|c|c|}
\hline \multicolumn{1}{|c|}{ Componentes CVL } & \multicolumn{2}{|c|}{ Estadístico t } \\
\hline Satisfacción por compasión & 1.66099 & 0.10053 \\
\hline Síndrome de quemado & 0.6106 & 0.54315 \\
\hline Estrés por trauma secundario & 0.4568 & 0.64902 \\
\hline
\end{tabular}

Fuente: Elaboración propia

En relación con el estado civil en la tabla 5 se muestran las medias y desviaciones estándar obtenidas. 
Revista Iberoamericana

de las Ciencias Sociales y Humanísticas

Tabla 5. Estadísticos por estado civil.

\begin{tabular}{|c|c|c|c|c|}
\hline \multirow{2}{*}{ Factores } & \multicolumn{2}{|l|}{ Estado Civil } & Media & $\begin{array}{c}\text { Desviación } \\
\text { estándar }\end{array}$ \\
\hline \multirow{3}{*}{ Satisfacción por compasión } & Soltero & 50.54467 & 10.661560 & \\
\cline { 2 - 5 } & Casado & 49.29617 & 9.475891 & \\
\cline { 2 - 5 } & Unión libre & 49.97573 & 9.437303 & \\
\hline \multirow{3}{*}{ Síndrome de quemado } & Soltero & 50.95661 & 9.466567 & \\
\cline { 2 - 5 } & Casado & 47.35257 & 9.197521 & \\
\cline { 2 - 5 } & Unión libre & 55.77884 & 13.582076 & \\
\hline \multirow{3}{*}{ Estrés por trauma secundario } & Soltero & 49.30878 & 10.161280 & \\
\cline { 2 - 5 } & Casado & 49.06370 & 8.905882 \\
\cline { 2 - 5 } & Unión libre & 57.57755 & 11.442600 & \\
\hline
\end{tabular}

Fuente: Elaboración propia

Como se puede apreciar en la tabla 6 esta variable no es un factor diferenciador para alguna de las escalas.

Tabla 6. Análisis por estado civil.

\begin{tabular}{|l|c|c|}
\hline \multicolumn{1}{|c|}{ Componentes CVL } & \multicolumn{2}{|c|}{ Estadístico f } \\
\hline Satisfacción por compasión & 0.14254 & 0.86737 \\
\hline Síndrome de quemado & 2.80604 & 0.06633 \\
\hline Estrés por trauma secundario & 2.64499 & 0.07713 \\
\hline
\end{tabular}

Fuente: Elaboración propia

En relación con la escolaridad en la tabla 7 se muestran las medias y desviaciones estándar obtenidas para cada subescala. 
Revista Iberoamericana

de las Ciencias Sociales y

Humanísticas

ISSN: $2395-7972$

Tabla 7. Estadísticos por escolaridad.

\begin{tabular}{|l|l|l|r|r|}
\hline \multicolumn{1}{|c|}{ Factores } & Escolaridad & Media & $\begin{array}{c}\text { Desviación } \\
\text { estándar }\end{array}$ \\
\cline { 1 - 5 } Satisfacción por compasión & Licenciatura & 48.32910 & 8.256455 & \\
\cline { 4 - 6 } & Carrera técnica & 50.74903 & 10.671033 & \\
\hline Síndrome de quemado & Licenciatura & 53.59821 & 7.646411 & \\
\cline { 2 - 5 } & Carrera técnica & 48.38701 & 10.555437 & \\
\hline Estrés por trauma secundario & Licenciatura & 51.40562 & 10.322325 & \\
\hline & Carrera técnica & 49.36990 & 9.878066 & \\
\hline
\end{tabular}

Fuente: Elaboración propia

Como se puede apreciar en la tabla 8 esta variable es un factor diferenciador para el síndrome de quemado.

Tabla 8. Análisis por escolaridad.

\begin{tabular}{|c|c|c|}
\hline Componentes CVL & Estadístico t & Valor $p$ \\
\hline Satisfacción por compasión & 1.02565 & 0.30807 \\
\hline Síndrome de quemado & -2.2621 & 0.02634 \\
\hline Estrés por trauma secundario & -0.8612 & 0.39164 \\
\hline
\end{tabular}

Fuente: Elaboración propia

\section{Análisis correlacional}

Se procedió a realizar los análisis correlacionales de edad, antigüedad y los años que el personal consideraba seguir trabajando. En las tablas 9,10 y 11 se puede apreciar que no hay correlación de ninguna de las tres variables con alguno de los componentes de la CVL. 
Revista Iberoamericana

de las Ciencias Sociales y

Humanísticas

Tabla 9. Análisis de correlación con la edad.

\begin{tabular}{|l|c|c|}
\hline \multicolumn{1}{|c|}{ Componentes CVL } & \multicolumn{1}{|c|}{$\begin{array}{c}\text { Edad } \\
\text { (Correlación) }\end{array}$} & Valor $p$ \\
\hline Satisfacción por compasión & 0.0210786 & 0.8490623 \\
\hline Síndrome de quemado & -0.100824 & 0.3614802 \\
\hline Estrés por trauma secundario & 0.1712410 & 0.1193609 \\
\hline
\end{tabular}

Fuente: Elaboración propia

Tabla 10. Análisis de correlación con los años laborando en enfermería.

\begin{tabular}{|l|c|c|}
\hline \multicolumn{1}{|c|}{ Componentes CVL } & \multicolumn{1}{|c|}{$\begin{array}{c}\text { Años lab. enf. } \\
\text { (Correlación) }\end{array}$} & Valores $p$ \\
\hline Satisfacción por compasión & 0.0536664 & 0.6277895 \\
\hline Síndrome de quemado & -0.071320 & 0.5191325 \\
\hline Estrés por trauma secundario & 0.1690619 & 0.1242077 \\
\hline
\end{tabular}

Fuente: Elaboración propia

Tabla 11. Análisis de correlación con los años futuros en enfermería.

\begin{tabular}{|l|c|c|}
\hline \multicolumn{1}{|c|}{ Componentes CVL } & \multicolumn{2}{|c|}{$\begin{array}{c}\text { Años futuros } \\
\text { en enfermería } \\
\text { (Correlación) }\end{array}$} \\
\hline Satisfacción por compasión & 0.1087521 & Valores $p$ \\
\hline Síndrome de quemado & 0.0774707 & 0.3247619 \\
\hline Estrés por trauma secundario & 0.0101982 & 0.4836490 \\
\hline
\end{tabular}

Fuente: Elaboración propia

Se calcularon las correlaciones lineales entre los componentes de la calidad de vida laboral, mediante el coeficiente de Pearson. Para su interpretación numérica se tomaron como referencia rangos previamente establecidos (James, Hastie, Tibshirani y Witten, 2017), en los que se consideran como "muy altas" las puntuaciones de 0.80 o mayores, como "altas" de 0.79 a 0.60 , "moderadas" de 0.59 a 0.40 , "bajas" de 0.39 a 0.20 y como "muy bajas" las menores de 0.20 , los resultados se presentan en la tabla 12 . 
Revista Iberoamericana

de las Ciencias Sociales y

Humanísticas

ISSN: $2395-7972$

La satisfacción por compasión presentó una correlación inversa moderada con el síndrome de quemado. Adicionalmente, el síndrome de quemado presentó una correlación directa baja con el estrés por trauma secundario.

Tabla 12. Coeficientes de correlación Pearson entre las variables analizadas.

\begin{tabular}{|c|c|c|c|c|}
\hline & & $\mathrm{SC}$ & SQ & ETS \\
\hline \multirow[t]{2}{*}{$\mathrm{SC}$} & Coef. & 1 & $\begin{array}{r}- \\
.421 *\end{array}$ & .120 \\
\hline & Sig. & & .000 & .279 \\
\hline \multirow[t]{2}{*}{ SQ } & Coef. & & 1 & $.264 *$ \\
\hline & Sig. & & & .015 \\
\hline \multirow[t]{3}{*}{ ETS } & Coef. & & & 1 \\
\hline & Sig. & & & \\
\hline & & & & \\
\hline
\end{tabular}

Fuente: Elaboración propia

\section{Discusión}

Como se mencionó anteriormente de los 84 profesionales que participaron en el estudio, 72 fueron mujeres y 12 hombres, equivalente en términos porcentuales a $85.7 \%$ de mujeres y $14.3 \%$ de hombres. Estos datos corroboran la presencia dominante del sexo femenino, como es característico de la profesión hasta la fecha, encontrándose ligeramente por arriba del porcentaje nacional de mujeres enfermeras que es de $85.0 \%$ (Instituto Nacional de Estadística y Geografía, INEGI, 2018). Así mismo se confirma lo encontrado por Barragán, Hernández y Peralta (2013) respecto de lo feminizado de esta profesión, lo cual según los autores genera un obstáculo para su avance a pesar de considerarse una profesión indispensable dentro del sistema de salud.

De acuerdo con la Encuesta Nacional de Ocupación y Empleo (INEGI, 2018) los profesionales en enfermería son más jóvenes con respecto a otras profesiones, la media de edad es de 36.8 años, en comparación con otros profesionales como la docencia (40.2 años) 


\section{Revista Iberoamericana \\ de las Ciencias Sociales y Humanísticas}

ISSN: $2395-7972$

y médicos (43.3 años) entre otras profesiones; esto se confirma en los datos encontrados, ya que la media aritmética de edad es de 33 años, siendo 3.8 años más jóvenes que el promedio nacional.

En cuanto al nivel educativo, se tiene que la media nacional a nivel licenciatura o grado más alto es del 10\%; los resultados del estudio arrojan que los profesionales con licenciatura en enfermería conforman casi la tercera parte (30.95\%), lo que los coloca por encima de la media nacional. Es importante mencionar que, en México el personal de enfermería es formado en distintos niveles educativos que van desde el básico, el técnico, medio superior, el superior y el posgrado, por ello no importa el grado académico obtenido, dichos profesionales pueden realizar actividades similares dentro de la institución hospitalaria.

En cuanto a los componentes de la calidad de vida laboral, se tiene que la satisfacción por compasión fue el componente que obtuvo la calificación más elevada $(\overline{\mathrm{x}}=43.01, \mathrm{~s}=4.91)$; además el $56 \%$ de los participantes manifestaron que casi siempre sienten satisfacción, el $50 \%$ orgullo, el $60.7 \%$ que disfrutan ayudar y el $72.6 \%$ que están felices con la profesión elegida, lo cual concuerda con la teoría de la conservación de los recursos de Hobfoll (1998), que establece que los profesionales de enfermería han desarrollado rasgos y habilidades enfocadas en los aspectos positivos del trabajo, ya que disfrutan ayudar y cuidar a los demás. De acuerdo con Buceta et al. (2019) el alto resultado de satisfacción por compasión aunado al bajo síndrome de quemado parece indicar que el personal del hospital estudiado es cercano del enfermo, ya que logra gestionar sus sentimientos lo que le permite no deshumanizar su atención.

A diferencia de los resultados de Aranda et al. (2015) que muestran que el desgaste ocupacional de los mexicanos se relaciona con las variables demográficas como el sexo, estado civil, escolaridad y otras, en el estudio realizado sólo la escolaridad resultó ser un factor diferenciador.

Los elevados puntajes de satisfacción por compasión y bajos de síndrome de quemado y estrés por trauma secundario significan, como señala Stamm (2010), que la mayor parte del personal se siente reforzado positivamente en su trabajo, sin una gran preocupación de ineficacia, ya sea como individuos o dentro de su organización. No sufren temor notable como resultado de su trabajo y por lo general pueden beneficiarse del compromiso, 
oportunidades de educación continua y otras oportunidades para crecer en su posición. Es probable que sean buenas influencias para sus colegas y su organización.

Desempeñarse en el área de servicios de salud no es fácil, ya que dichos profesionales están expuestos a situaciones de crisis y desgaste emocional y es bien conocido que la satisfacción y el bienestar laboral influyen tanto en la calidad de los servicios como en el comportamiento efectivo de los profesionales; por ello conocer y adaptar el sistema de salud a las necesidades reales de la población y los profesionales puede ayudar a incrementar el sentido de satisfacción y bienestar personal de estos, lo que redundará en una mayor satisfacción en los pacientes.

Una de las limitaciones del estudio es que abarcó un sólo hospital.

\section{Conclusión}

Del análisis de los datos sociodemográficos se puede concluir que tanto por edad como por sexo la muestra tiene una conformación similar a los datos a nivel nacional. No así en cuanto al nivel educativo que es mayor en el personal estudiado.

Considerando los puntos de corte los resultados son positivos ya que 53 personas se encuentran con alta satisfacción por compasión en tanto que 60 y 71 tienen bajo síndrome de quemado y estrés por trauma secundario respectivamente.

En cuanto a la calidad de vida laboral los resultados obtenidos fueron favorables, ya que, de los tres componentes de la calidad de vida laboral, la satisfacción por compasión fue elevada mientras que el síndrome de quemado y el estrés por trauma secundario estuvieron en niveles bajos, lo que permite identificar condiciones adecuadas. Considerando los cinco niveles de categorías de CVL se puede decir que el $63.1 \%$ del personal se encuentra reforzado positivamente.

Como resultado de los análisis inferenciales correspondientes a las escalas con relación a los datos sociodemográficos sexo, estado civil y escolaridad se concluye que solo esta última es un factor diferenciador para el síndrome de quemado.

De los resultados de los análisis correlacionales de los datos sociodemográficos de edad, antigüedad y los años que el personal consideraba seguir trabajando se concluye que no hay correlación de ninguna de ellos con alguno de los componentes de la CVL. 
Adicionalmente se encontró una relación inversa moderada entre la satisfacción por compasión y el síndrome de quemado lo cual da cuenta que la satisfacción puede provenir de contar con apoyo y recursos, así como del desarrollo de estrategias efectivas para manejar el síndrome de quemado.

Así mismo se encontró, concordante con la literatura, que el síndrome de quemado tiene una relación directa con el estrés por trauma secundario, aunque ésta fue muy baja en el hospital estudiado.

Se considera importante diversificar el campo de estudio para futuras investigaciones, en el hospital estudiado hacer una segunda fase cualitativa, incluir hospitales públicos y privados, hacerlo extensivo a otros estados de la república, ya que si bien los resultados obtenidos en el estudio que aquí se presenta fueron positivos, es importante conocer la realidad del sector por la importancia que tiene no solo para las instituciones y los prestadores de servicios de salud en cuanto al manejo de estrategias para incrementar y mantener la calidad de vida laboral, sino también por el impacto que ello tiene en los pacientes mismos. 
Revista Iberoamericana

de las Ciencias Sociales y

Humanísticas

ISSN: $2395-7972$

\section{Referencias}

Aranda, C., Pando, M. y Salazar, J. (2015). Síndrome de Burnout en trabajadores de diversas actividades económicas en México. Revista Iberoamericana de Psicología: Ciencia y Tecnología, 8(2), 23-28. Recuperado de Dialnet-

SindromeDeBurnoutEnTrabajadoresDeDiversasActividad-5608873 (1).pdf

Armayones, M. (2010). Técnicas de apoyo psicológico y social en situaciones de crisis. Como desarrollar las habilidades adecuadas ante situaciones de emergencia. España: Ideaspropias Editorial S.L.

Barragán, O., Hernández, E. y Peralta, S. (2013). Una mirada a la profesión de enfermería desde la perspectiva de género. Epistemus, (15) 34-37. Recuperado de https://biblat.unam.mx/hevila/EpistemusCienciatecnologiaysalud/2013/no15/5.pdf

Bernal, C. (2010). Metodología de la investigación. Colombia: Pearson Educación.

Bride, B., Radey, M. y Figley, Cr. (August, 2007). Measuring Compassion Fatigue. Clinical Social Work Journal, 35, 155 - 163. doi: 155-163. 10.1007/s10615-0070091-7.

Buceta, M. I., Bermejo, J. C., y Villacieros, M. (2019). Elementos potenciadores de la satisfacción por compasión en profesionales sociosanitarios. Anales de Psicología, 35(2), 323-331. doi: 10.6018/analesps.35.2.345101

Dall’occhio M. y Lermoli R. (2012). El síndrome del Quemado. Centro de Estudios del Estrés y la Ansiedad Burnout. Publicado el 20 de agosto del 2012. Recuperado de www.psicoadolescencia.com.ar

Díaz, M., Stimolo, M. y Caro, N. (2010). Satisfacción Laboral y Síndrome de Desgaste Laboral en Enfermeros de Hospitales Públicos Córdoba, Argentina. Medicina y Seguridad del Trabajo, 56(218), 22-38. Recuperado de http://scielo.isciii.es/pdf/mesetra/v56n218/original2.pdf

Fundación Europea para el Mejoramiento de las Condiciones de Vida (2002). New work organization, working conditions and quality of work: towards the flexible firm. Recuperado de http://edz.bib.uni-mannheim.de/daten/edz-ma/esl/02/ef0274en.pdf

Figley, C. R. (1995). Compassion fatigue: Coping with secondary traumatic stress disorder. New York: Brunner/Mazel. 
Flores, N., Jenaro, C., Cruz, M., Vega, V. y Pérez, M. C. (2013). Síndrome de burnout y calidad de vida laboral en profesionales de servicios sanitarios. Pensando Psicología, 9(16), 7-21.

Hanzeliková, A., García, M.V., Pomares, M., Pardo, M.J. y Del Monte, J. (2011). La calidad de vida profesional de las enfermeras en geriatria. Enfermería Global, 10(24). doi: 10.4321/S1695-61412011000400012.

Hobfoll, S. (1998). The psychology and philosophy of stress, culture and community. New York: Plenum.

Hobfoll, S. (2001). The influence of culture, community, and the nested! self in the stress process: advancing conservation of resources theory. Applied Psychology, 50(3), $337-421$.

Hurtado, D. y Pereira, F. (2012). El síndrome de desgaste profesional (burnout syndrome): manifestación de la ruptura de reciprocidad laboral. Revista Salud Bosque, 2(2), 2938. Recuperado de https://www.researchgate.net/publication/237396662_El_sindrome_de_desgaste_pr ofesional_Burnout_Syndrome_manifestacion_de_la_ruptura_de_reciprocidad_labor $\mathrm{al} / \mathrm{stats}$

INEGI (2018, febrero). Encuesta Nacional de Ocupación y Empleo (ENOE). Retrived from población de 15 años y más de edad. Recuperado de http://www.beta.inegi.org.mx/proyectos/enchogares/regulares/enoe/

James, G., Hastie, T., Tibshirani, R. y Witten, D. (2017). An introduction to Statistical learning: With application in R. New York: Springer-Verlag.

Kulkarni, P. (2013). A literature review on training y development and quality of work life. International Refereed Research Journal, IV(2), 136 - 143.

Lloyd, C., King, R. y Chenoweth, L. (2002). Social work, stress and burnout: A review. Journal of Mental Health, 11(3), 255-266. doi: 10.1080/09638230020023642

Marín-Tejeda, M. (2017). Prevención de burnout y fatiga por compasión: evaluación de una intervención grupal. Journal of Behavior, Health \& Social Issues, 9(2),117-123. Recuperado de https://www.redalyc.org/articulo.oa?id=2822/282255144012 
Revista Iberoamericana

de las Ciencias Sociales y Humanísticas

ISSN: $2395-7972$

Meda, R, Moreno-Jiménez, B, Palomera, A, Arias, E, y Vargas, R. (2012). La Evaluación del Estrés Traumático Secundario: Estudio Comparado en Bomberos y Paramédicos de los Servicios de Emergencia de Guadalajara, México. Terapia psicológica, 30(2), 31-41. doi: 10.4067/S0718-48082012000200003

Mendoza, I., García, B., y Serna, H. (2014, septiembre). Validación de un instrumento de calidad de vida laboral en el contexto mexicano. Recuperado de https://www.researchgate.net/publication/265551688

Munroe, J. F. (1999). Ethical issues associated with secondary trauma in therapists. En H. Stamm (Ed.) Secondary traumatic stress: Self-care issues for clinicians, researchers and educators (pp. 211-229). MD: Sidran Press.

Organización para la Cooperación y el Desarrollo Económico (OCDE, 2019). OECD Employment Outlook 2019: The Future of Work. Paris: OECD Publishing. doi.org/10.1787/9ee00155-en.

Organización Mundial de la Salud. (OMS, 2015). Enfermería. Recuperado de //www.who.int/topics/nursing/es/

Pooler, D. K., Wolfer, T., y Freeman, M. (2014). Finding joy in social work: Interpersonal sources. Families in Society, 95, 35-42. doi:10.1606/1044-3894.2014.95.5

Pryce, D., Schackelford, K. y Pryce, G. (2007). Secondary Traumatic Stress and the Child Welfare Professional. Iowa, USA: Follmer Group, T.H.E.

Secretaría del Trabajo y Previsión Social (2017). Seguridad y salud en el trabajo en México: Avances, retos y desafíos. México, Cdmx: Subsecretaria de Previsión Social.

Segurado, A., y Agulló, E. (2002). Calidad de vida laboral: hacia un enfoque integrador desde la Psicología Social. Psicothema, 14(4), 828-836.

Sibbald, B., Enzer, I., Cooper, C., Rout, U., \& Sutherland, V. (2000). Job satisfaction in 1987, 1990 and 1998: Lessons for the future? Family Practice, 17, 364-371.

Stamm B. (2010). The Concise ProQOL Manual. Recuperado de http://www.proqol.org/uploads/ProQOL_Concise_2ndEd_12-2010.pdf

Villavicencio-Ayub (2019). Trastornos psicosomáticos, emocionales y físicos, condiciones de malas condiciones laborales. Recuperado de https://www.dgcs.unam.mx/boletin/bdboletin/2019_295.html 


\section{Revista Iberoamericana de las Ciencias Sociales y Humanísticas}

WHOQOL Group (1998). Development of the World Health Organization WHOQOLBREF Quality of Life Assessment. Psychological Medicine, 28, 551-558.

\begin{tabular}{|c|c|}
\hline Rol de Contribución & Autor(es) \\
\hline Conceptualización & $\begin{array}{l}\text { Ruth Noemí Ojeda López (Principal) Jennifer Mul Encalada } \\
\text { (Igual) }\end{array}$ \\
\hline Metodología & $\begin{array}{l}\text { Ruth Noemí Ojeda López (Principal) Jennifer Mul Encalada } \\
\text { (Igual) }\end{array}$ \\
\hline Software & NO APLICA \\
\hline Validación & Jennifer Mul Encalada \\
\hline Análisis Formal & $\begin{array}{l}\text { Ruth Noemí Ojeda López (Principal) Jennifer Mul Encalada } \\
\text { (Igual) }\end{array}$ \\
\hline Investigación & $\begin{array}{l}\text { Ruth Noemí Ojeda López (Principal) Jennifer Mul Encalada } \\
\text { (Igual) y Olivia Jiménez Díez (que apoya) }\end{array}$ \\
\hline Recursos & Ruth Noemí Ojeda López \\
\hline Curación de datos & Jennifer Mul Encalada. \\
\hline $\begin{array}{l}\text { Escritura - Preparación del } \\
\text { borrador original }\end{array}$ & $\begin{array}{l}\text { Ruth Noemí Ojeda López (Principal) Olivia Jiménez Díez } \\
\text { (que apoya). }\end{array}$ \\
\hline $\begin{array}{l}\text { Escritura - Revisión y } \\
\text { edición }\end{array}$ & $\begin{array}{l}\text { Ruth Noemí Ojeda López (Principal) Jennifer Mul Encalada } \\
\text { (Igual) }\end{array}$ \\
\hline Visualización & Olivia Jiménez Diez. \\
\hline Supervisión & Ruth Noemí Ojeda López \\
\hline $\begin{array}{l}\text { Administración de } \\
\text { Proyectos }\end{array}$ & Ruth Noemí Ojeda López \\
\hline Adquisición de fondos & Ruht Noemí Ojeda López \\
\hline
\end{tabular}

